\title{
An Unusual Case of Nosocomial Trichosporon asahii Fungemia in a Patient with Tuberculous meningitis
}

\author{
Vijaya S Rajmane, ${ }^{1,}$ Shivkumar T Rajmane, ${ }^{2}$ Virendra C Patil, ${ }^{3}$ and Vinayak V Raje ${ }^{4}$ \\ ${ }^{1}$ Department of Microbiology, Krishna Institute of Medical Sciences University, Karad, India \\ ${ }^{2}$ Department of Orthopedics, Institute of Medical Sciences and Research, Mayani, India \\ ${ }^{3}$ Department of Medicine, Krishna Institute of Medical Sciences University, Karad, India \\ ${ }^{4}$ Department of Neurosurgery, Krishna Institute of Medical Sciences University, Karad, India \\ Corresponding author: Vijaya S Rajmane, Department of Microbiology, Krishna Institute of Medical Sciences University, Karad, India. Tel: +91-9860299944, Fax: +91-2164243272, \\ E-mail: drvsrajmane@yahoo.com
}

Received 2016 July 08; Revised 2017 April 23; Accepted 2017 May 01.

\begin{abstract}
Introduction: Invasive Trichosporon species have been documented mostly in neutropenic patients with underlying hematological malignancies with high mortality, and in critically ill patients exposed to multiple invasive medical procedures. This fungus has been recognized as the second or third most common agent of yeast fungemia.

Case Presentation: This study reports on invasive infection with Trichosporon asahii in a non-neutropenic patient with Tuberculous meningitis, who was a known case of diabetes mellitus type II.

Conclusions: Although fungemia due to Trichosporon species is an opportunistic pathogen in granulocytopenic patients, there is a steady increase in the number of such cases in non-neutropenic and non-hematological malignant patients. Thus, clinicians as well as microbiologists should be aware of such infections in critically ill patients as early diagnosis and timely management with appropriate antifungal drugs could decrease morbidity and mortality rate.
\end{abstract}

Keywords: Fungemia, Antifungal Agents, Trichosporon asahii

\section{Introduction}

Over the past 2 decades, invasive mycoses due to emerging fungal pathogens have increased significantly and are usually associated with high mortality rate (1). Trichosporon species is a basidiomycete that may be part of the normal flora in the gastrointestinal tract and transiently colonize the skin, nails, and respiratory tract in humans $(2,3)$. Trichosporon is a known cause of white piedra in immunocompetent hosts (4). Invasive infection has emerged as an opportunistic infection in immunocompromised hosts with hematological malignancies, solid organ tumors, burn patients, patients with human immunodeficiency virus (HIV) infection, end stage renal disease, and recipients of prosthetic heart valves (2). Trichosporon asahii, the most common of the Trichosporon species in systemic Trichosporon infection, is life threatening due to decreased susceptibility to amphotericin B, especially in severely granulocytopenic patients with underlying hematological malignancy $(5,6)$. This study reports on a case of fungemia due to Trichosporon asahii in a non-neutropenic patient with Tuberculous meningitis with diabetes mellitus.

\section{Case Presentation}

A 72-year-old female presented with fever, altered sensorium, and seizure (new onset) for 1 day was admitted to Krishna hospital, 1100 bedded private hospital attached to a medical college; krishna institute of medical sciences and research, Deemed University, Karad, India. The patient had severe headache, fatigue, and malaise one week prior to admission in the hospital. She was a known case of type 2 diabetes mellitus for the last 15 years and was on oral hypoglycemic drugs, which was uncontrolled. There was no history of vomiting. On general examination, the patient was averagely built, drowsy, with mild pallor, pulse rate of $96 /$ minute, blood pressure of $130 / 80 \mathrm{~mm} \mathrm{Hg}$, and respiratory rate of $24 /$ minute. Other vital parameters were within normal limits.

The patient was immediately shifted to the medical intensive care unit, intubated, and the intravenous catheter was inserted and anticonvulsant drugs, injection of monocef and vancomycin were initiated. Blood investigations were as follows: Hemoglobin $(\mathrm{Hb})=10.8$ gram percentage, total white blood count $(\mathrm{WBC})$ count $=9800 \mathrm{~mm}^{3}$, platelet 
count $=1.8$ lacs $/ \mathrm{mm}^{3}$, blood sugar level $(\mathrm{BSL})($ random $)=$ $210 \mathrm{mg} / \mathrm{dL}$, blood urea level $=36 \mathrm{mg} / \mathrm{dL}$, serum creatinine $=1.3 \mathrm{mg} / \mathrm{dL}$, serum $\mathrm{Na}^{+}=142 \mathrm{meq} / \mathrm{L}$, and serum $\mathrm{K}^{+}=4.2$ meq/L. Liver function tests were normal. Peripheral smear was negative for malarial parasite. Test for human immunodeficiency virus (HIV), HBsAg, and hepatitis C virus (HCV) were non-reactive. Urine analysis revealed mild ketone bodies. The electrocardiogram (ECG) showed no significant ST-T changes, and chest X-ray (PA view) was within normal limits. Plain CT brain showed signs of meningitis. The CSF cytology was as follows: 180 cells $/ \mathrm{mm}^{3}$ with lymphocytic predominance (80\%), CSF proteins $=130 \mathrm{mg} \%$, and CSF sugar $=84 \mathrm{mg} \%$. Gram stain of CSF showed few pus cells but no microorganisms. Ziehl Neelsen stain of CSF showed acid fast bacilli. Bacterial and fungal cultures were sterile. The CSF was taken on Lowenstein Jensen medium for Mycobacterial culture. Blood culture was sterile for both bacterial and fungal pathogens. Sputum was negative for acid fast bacilli.

The patient was diagnosed as a case of Tuberculous meningitis and was started on anti-tubercular drug therapy. The patient was responding to treatment and recovered from fever as well as signs and symptoms of meningitis. The intravenous catheter was removed and discarded after 2 weeks as the patient was found to be in a good condition. The catheter tip was not processed for any microbiological culture. Suddenly, on the 8th day of admission, the patient had sudden onset of high grade fever without chills and not subsiding with antipyretics. On the 3rd day of fever, blood was collected in Biphasic medium containing brain heart infusion agar with broth for culture.

The blood culture revealed growth of yeast-like colonies after 72 hours of incubation at $30^{\circ} \mathrm{C}$. Colonies were 1 to $2 \mathrm{~mm}$ and white; profusely wrinkled cerebriform growth heaped up in the center with irregular margins. Gram stain showed 3 to $4 \mu \mathrm{m}$ gram positive oval yeast cells arranged in chains. Germ tube test was negative, urease test positive, and morphology on corn meal agar showed budding of cells with barrel-shaped arthroconidia and absent lateral conidia (Figure 1). Based upon these findings, the isolate was identified as Trichosporon species. The isolate was sent to postgraduate institute of medical education and research (PGIMER), Chandigarh, India, which is the only world health organization (WHO) collaborating center for reference and research on fungi of medical importance in the world. The isolate was identified and confirmed to be T. asahii by both phenotypic as well as genotypic methods on the basis of Ribosomal DNA Intergenic Spacer 1 sequencing.

The report was immediately dispatched and the patient was started on IV fluconazole until the antifungal susceptibility test was carried out. Antifungal susceptibility

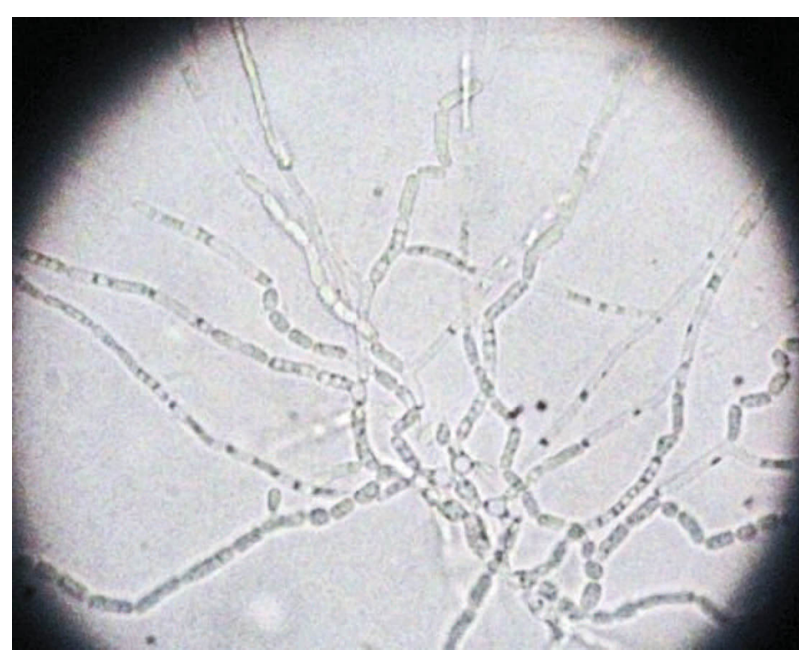

Figure 1. Cornmeal agar Showing Budding Cells with Barrel-Shaped Arthroconidia and Absent Lateral Conidia

testing was carried out by broth microdilution method, according to the clinical and laboratory standards institute (CLSI) M27-A3 guidelines (7). The minimum inhibitory concentrations (MICs) of the isolates tested were $2 \mu \mathrm{g} / \mathrm{mL}$ for amphotericin B and $32 \mu \mathrm{g} / \mathrm{mL}$ for flucytosine, which were consistently high; intermediate for itraconazole i.e. $0.5 \mu \mathrm{g} / \mathrm{mL} ; 4 \mu \mathrm{g} / \mathrm{mL}$ for fluconazole and $0.125 \mu \mathrm{g} / \mathrm{mL}$ for voriconazole, which were very low showing good in vitro susceptibility after 48 hours of incubation.

The patient continued receiving IV fluconazole at a dose of $200 \mathrm{mg}$ twice a day. The patient responded well and fever subsided after 4 to 5 days of therapy. The patient was shifted to oral fluconazole at a dose of $200 \mathrm{mg}$ once daily, which was continued for 8 weeks. Blood culture was repeated after 2 weeks of therapy and was sterile. The patient was discharged in ambulatory state with continuation of anti-tubercular drugs and was advised regular follow up.

\section{Discussion}

Trichosporonosis is recognized as one of the emerging opportunistic mycosis in tertiary care hospitals, worldwide $(8,9)$. These non-Candida yeasts present varied clinical manifestations ranging from superficial infections to severe life threatening invasive diseases in the immunocompromised host (10). Trichosporon asahii fungemia is insidious in onset and presents a diagnostic challenge, especially in developing countries, due to lack of awareness as well as significant diagnostic features of this pathogen (4). Though invasive Trichosporon infections have been most commonly reported in patients with hematological malig- 
nancies, they occur in non-cancer patients with chronic illness and disruption of skin and mucous membranes (1).

Fungemia due to Trichosporon species is frequently fatal despite antifungal therapy due to its varied susceptibility to amphotericin B and 5-flurocytosine (2). Fluconazole, voriconazole, and newer triazoles, namely posaconazole and ravuconazole have shown potent in vitro activity and are currently the drug of choice against $T$. asahii and other Trichosporon species $(2,6)$. Echinocandins are reported to be ineffective against this pathogen. There are very few reports available on the susceptibility of T. asahii to antifungal drugs as in vitro susceptibility methods are not standardized and MIC breakpoints for Trichosporon species have not yet been determined (2).

The present study reports on a case of tuberculous meningitis with known diabetes mellitus admitted to the intensive care unit. This patient did not have neutropenia nor any malignancy. The presence of uncontrolled diabetes mellitus, prolonged ICU stay, and IV catheter in situ were few of the various risk factors, to which the patient was exposed. Thus, the only port of entrance for T. asahii in the bloodstream is most likely to be percutaneously inserted IV catheter via the colonized skin. A relationship between Trichosporonosis and having undergone an invasive clinical procedure has been well established (11). Kontoyiannis et al. and Ruan et al. have reported cases of invasive trichosporonosis related to the use of central venous catheters thus leading to exogenous acquisition of this pathogen $(12,13)$. The duration of treatment of Trichosporon fungemia depends on the patient's condition. A few studies have continued treatment till subsequent blood cultures are negative followed by oral antifungal agents for the next few weeks. If the patient is neutropenic, then prophylactic treatment is followed in spite of negative blood culture till the neutrophil counts are normal (14).

This study showed good in vitro susceptibility of fluconazole and voriconazole against $T$. asahii and the patient responded well to the given treatment without any fatal consequences.

\subsection{Conclusion}

With the establishment of the pathogenic role of $T$. asahii even in non-neutropenic and non-cancer patients, this study suggests that clinicians should remain aware of invasive infections by this pathogen in clinically deteriorated patients exposed to various risk factors. This demands invasive mycological investigations for early identification and timely institution of appropriate antifungal therapy along with elimination of predisposing factors for favorable outcome of the patient.

\section{Footnote}

Financial Disclosure: The authors declare that they had no financial interests related to the material in the manuscript.

\section{References}

1. Chagas-Neto TC, Chaves GM, Melo AS, Colombo AL. Bloodstream infections due to Trichosporon spp.: species distribution, Trichosporon asahii genotypes determined on the basis of ribosomal DNA intergenic spacer 1 sequencing, and antifungal susceptibility testing. $J$ Clin Microbiol. 2009;47(4):1074-81. doi:10.1128/JCM.01614-08. [PubMed: 19225102]

2. Karabay O, Madariaga MG, Kocoglu E, Ince N, Kandirali E. Trichosporon asahii fungemia in a patient with non-hematological malignancy. Jpn J Infect Dis. 2006;59(2):129-31. [PubMed: 16632916].

3. Hashino S, Takahashi S, Morita R, Kanamori H, Onozawa M, Kawamura T, et al. Fungemia due to Trichosporon dermatis in a patient with refractory Burkitt's leukemia. Blood Res. 2013;48(2):154-6. doi: 10.5045/br.2013.48.2.154. [PubMed: 23826589].

4. Chowdhary A, Ahmad S, Khan ZU, Doval DC, Randhawa HS. Trichosporon asahii as an emerging etiological agent of disseminated trichosporonosis: A case report and an update. IJMM. 2004;22(1):1622.

5. Asada N, Uryu H, Koseki M, Takeuchi M, Komatsu M, Matsue K. Successful treatment of breakthrough Trichosporon asahii fungemia with voriconazole in a patient with acute myeloid leukemia. Clin Infect Dis. 2006;43(4):e39-41. doi: 10.1086/505970. [PubMed: 16838224].

6. Shih-Ta S, Ya-Sung Y, Ming-Yieh P. Nosocomial trichosporon asahii fungemia in a patient with secondary hemochromatosis: A rare case report. J Microbiol Immunol Infect. 2010;43(1):77-80.

7. CLSI . Reference method for broth dilution antifungal susceptibility testing ofyeasts: Approved standard-edition. Third ed. Wayne, PA, USA: Clinical and Laboratory Standards Institute; 2008.

8. Gunn SR, Reveles XT, Hamlington JD, Sadkowski LC, Johnson-Pais TL, Jorgensen JH. Use of DNA sequencing analysis to confirm fungemia due to Trichosporon dermatis in a pediatric patient. J Clin Microbiol. 2006;44(3):1175-7. doi: 10.1128/JCM.44.3.1175-1177.2006. [PubMed: 16517924].

9. Colombo AL, Padovan AC, Chaves GM. Current knowledge of Trichosporon spp. and Trichosporonosis. Clin Microbiol Rev. 2011;24(4):682-700. doi: 10.1128/CMR.00003-11. [PubMed: 21976604].

10. O'Gorman C, McMullan R, Webb CH, Bedi A. Trichosporon asahii. Blood-stream infection in a non-cancer patient receiving combination antifungal therapy. Ulster Med J. 2006;75(3):226-7. [PubMed: 16964817].

11. Montoyo AM, Gonzalez GM. Trichosporon spp.: an emerging fungal pathogen. Med Univ. 2014;16(62):37-43.

12. Kontoyiannis DP, Torres HA, Chagua M, Hachem R, Tarrand JJ, Bodey GP, et al. Trichosporonosis in a tertiary care cancer center: risk factors, changing spectrum and determinants of outcome. Scand J InfectDis. 2004;36(8):564-9. doi:10.1080/00365540410017563. [PubMed: 15370667].

13. Ruan SY, Chien JY, Hsueh PR. Invasive trichosporonosis caused by Trichosporon asahii and other unusual Trichosporon species at a medical center in Taiwan. Clin Infect Dis. 2009;49(1):e11-7. doi: 10.1086/599614. [PubMed: 19489711].

14. Hikmet Gulsah T, Sule T. Mehmet onur candir and gurses sahin. Two case presentations infected by trichosporon asahii and treated with voriconazole successfully. Infect Dis. 2015:3. 